\title{
LA PHILOSOPHIE COMME MÉTHODOLOGIE: LA CONCEPTION SCEPTICO-RATIONALISTE DE LA RAISON CHEZ BAYLE
}

\author{
Kristen Irwin ${ }^{*}$ \\ kirwin@ucsd.edu
}

RESUMO Bayle é frequentemente considerado um cético, mas sua concepção da razão nem sempre é clara. O que é, ao contrário, claro, é que ele manifesta uma profunda desconfiança no que concerne a capacidade da razão de produzir um conhecimento certo. Entretanto, uma nova concepção de Bayle como "racionalista estratoniciano » foi desenvolvida por Gianluca Mori, que oferece uma descrição detalhada de Bayle como um filósofo crítico desejoso de explicar todas as posições possivveis em sua complexidade e de tirar todas as consequencias dos argumentos invocados ao serviço de princípios chamados " estratonicianos ». Esta concepção de Bayle como racionalista crítico estratoniciano torna possível uma interpretação de Bayle menos como um "super cético » à maneira de Richard Popkin do que como alguém que permite a razão operar de forma mais ampla e com mais autoridade do que permitiria um ceticismo. Mori afirma que a concepção bayleana da razão é bem forte. Para ele a razão pode chegar até o fim, com autoridade absoluta, e retirar conclusões. Eu mantenho que a concepção de Bayle da natureza e da função da razão se situa entre o racionalismo estratoniciano de Mori e a interpretação "supercética » de Popkin. Como Mori, penso que se trata de um racionalismo " crítico ", mas contra ele penso que a razão mesma define e recomenda os seus próprios limites. Chamo esta concepção da razão de

* University of California, San Diego / Biola University, USA. Recebido em 03/08/2009 e aceito em 17/09/2009.

KRITERION, Belo Horizonte, nº 120, Dez./2009, p. 363-376. 
" cético-racionalista » e, seguindo Bayle, sugiro como o «bom senso » (que faz parte da razão) ajuda a definir estes limites.

Palavras-chave racionalismo cético, racionalismo estratoniciano, retorsão

RÉSUMÉ Bayle est souvent considéré comme sceptique, mais sa conception de la raison n'est pas toujours claire; ce qui en revanche est clair, c'est qu'il manifeste une profonde méfiance à l'égard des capacités de la raison de livrer une connaissance certaine. Cependant, une nouvelle interprétation de Bayle comme rationaliste "stratonicien 》 a été développée par Gianluca Mori, qui donne une description détaillée de Bayle comme philosophe critique désireux de rendre compte de toutes positions possibles dans leur complexité et de tirer toutes les conséquences des arguments invoqués au service des principes dits « stratoniciens ». Cette conception de Bayle comme rationaliste critique stratonicien rend possible une interprétation de Bayle moins comme un "supersceptique » à la Richard Popkin, que celui qui permet à la raison d'opérer avec une portée et une autorité plus grandes que ne le permettrait le scepticisme. Mori affirme que la conception de la raison chez Bayle est assez forte; selon lui, la raison peut aller jusqu'au bout, avec une autorité absolue, et tirer des conclusions. Je soutiens que la conception de la nature et de la fonction de la raison chez Bayle se situe en fait entre le rationalisme stratonicien de Mori et l'interprétation « supersceptique » de Popkin. Comme Mori, je pense qu'il s'agit d'un rationalisme "critique "; mais contre lui, je pense que la raison elle-même définit et recommande ses propres limites. J'appelle cette conception de la raison "sceptico-rationaliste " et, en suivant Bayle, je suggère comment le «bon sens» (qui fait partie de la raison) aide à définir ces limites.

Mots clés sceptico-rationalisme, rationalisme stratonicienne, retórsion, bon sens

S'il ne fallait retenir qu'un terme pour caractériser la position philosophique de Pierre Bayle, le choix serait évident : le scepticisme. La tradition herméneutique considérant Bayle comme un sceptique a commencé avec ses contemporains et se prolonge avec les commentateurs actuels comme Richard Popkin et Thomas Lennon. Il est évident que Bayle manifeste une profonde méfiance à l'égard de ceux qui croient aux capacités de la raison 
de livrer une connaissance certaine. Selon lui, la raison est conduite à sa propre ruine si elle n'est pas limitée. Cependant avec la publication de Bayle philosophe par Gianluca Mori, une nouvelle interprétation s'est fait jour : celle d'un Bayle rationaliste « stratonicien ». Ce nouveau point de repère donne une interprétation détaillée de Bayle comme philosophe critique désireux de rendre compte de toutes les positions possibles dans leur complexité et de tirer toutes les conséquences des arguments invoqués. Cette conception de Bayle comme rationaliste critique stratonicien rend possible une interprétation de Bayle moins comme un "supersceptique » à la Richard Popkin, que celui qui permet à la raison d'opérer avec une portée et une autorité plus grandes que ne le permettait le scepticisme. Mori soutient que la conception de la raison chez Bayle est assez forte ; selon lui, la raison peut aller jusqu'au bout, avec une autorité absolue, et tirer des conclusions. Je soutiens que la conception de la nature et de la fonction de la raison chez Bayle se situe en fait entre le rationalisme stratonicien de Mori et l'interprétation « supersceptique » de Popkin. Je pense, comme Mori, qu'il s'agit d'un rationalisme « critique »; mais contre lui, je pense que la raison elle-même définit et recommande ses propres limites.

\section{I - La conception « supersceptique » de la raison}

L'interprétation classique de la conception de la raison chez Bayle est celle d'un sceptique du XVIIe siècle : la raison paraît être utile dans la mesure où elle nous donne la capacité de tirer des conclusions, mais elle se heurte à tant de contradictions et produit tant de paradoxes qu'en fin de compte elle se sape, en sorte que l'on ne peut pas lui faire confiance. Selon une logique fidéiste simpliste, on dira : puisqu'on ne peut pas faire confiance à la raison pour nous guider vers la vérité, il faut chercher un autre guide plus fiable - la foi ou la révélation.

Pierre-Daniel Huet (1630-1721) peut être considéré comme l'avocat le plus représentatif de cette position au XVIIe siècle. Il fournit un modèle utile pour comprendre cette interprétation de Bayle. Dans son Traité philosophique de la faiblesse de l'esprit humain, paru en 1723, Huet donne un résumé succinct de son fidéisme sceptique. Sa méfiance à l'égard de la raison est évidente :

Mais Dieu par sa bonté répare ce défaut de la nature humaine, en nous accordant ce don inestimable de la foi, qui confirme la raison chancellante, \& corrige cet embarras des doutes qu'il faut apporter à la connoissance des choses. Car, par exemple, ma raison ne pouvant me faire connoître avec une entière évidence, \& une parfaite certitude, s'il 
y a des corps, quelle est l'origine du monde, \& plusieurs autres choses pareilles, après que j'ai reçu la foi ; tous ces doutes s'évanouissent [...] De même donc que dans les choses de la foi, la foi vient au secours de la raison chancellante ; elle nous aide aussi dans toutes les autres choses que nous connoissons par la raison, pour nous rassurer dans nos doutes, \& pour rétablir la raison dans ses droits, dont elle était déchue ; c'est-à-dire, dans la connoissance de la verité, qu'elle desire naturellement. ${ }^{1}$

Huet ne rejette pas complètement la raison comme autorité fiable épistémique, mais il la présente comme " défectueuse », " chancellante » et " déchue ». La nature humaine est " défectueuse ", sans doute à cause de l'instabilité de la raison et de son incapacité à assurer une « entière évidence » et une « parfaite certitude ». Huet n'affirme pas simplement que la raison manque de certitude, mais aussi qu'il faut sans cesse douter de ce qu'elle enseigne. Dans le deuxième livre du Traité - sur « la plus sure, \& la plus légitime voye de philosopher »-, il soutient précisément que la raison est une maîtresse douteuse :

Nous avons principalement une grande attention à ne rien admettre qui soit contraire à la foi révélée, tenant pour très-certain \& indubitable ce que Dieu a marquée dans notre ame par la foi, guide $\&$ maîtresse de la raison ; \& tenant pour douteux tout ce que la raison nous enseigne. ${ }^{2}$

Huet se demande si la raison est capable de nous enseigner quoi que ce soit de véritable ; il faut douter de tout ce que la raison nous enseigne. Il semble qu'il n'y ait pas de place dans cette conception de la raison pour une fonction positive, et Richard Popkin identifie cette position comme une espèce de « pyrrhonisme chrétien ». L'interprétation «supersceptique» consiste, pour Popkin, à soutenir que, chez Bayle, la conception de la raison ressemble par plusieurs traits à celle de Huet, mais qu'elle est plus radicale :

In each case, Bayle is not solely or merely concerned to challenge a theory but to use the occasion to generalize an attack to all theories and to show the hopeless abysses to which all human intellectual endeavors lead... ${ }^{3}$

Entre autres passages, Popkin fonde son interprétation sur les célèbres remarques du Dictionnaire à propos de la nature autodestructrice de la raison.

1 Huet, Pierre-Daniel. Traité philosophique de la faiblesse de l'esprit humain. Amsterdam : H. du Sauzet, 1723, Livre II, ch. II ; p. 182-3, 187.

2 Livre II, ch. IX; p. 216-7.

3 Richard H. The History of Scepticism : From Savonarola to Bayle. Oxford : Oxford University Press, 2003, p. 289. 
Dans une remarque de l'article « Bunel (Pierre) », Bayle utilise l'analogie pittoresque de Pénélope pour montrer à quel point la raison se défait :

Je suis ravi qu'un tel auteur [Reginald Polus] me fournisse de quoi confirmer ce que j'établis en divers endroits, que notre raison n'est propre qu'à brouiller tout, \& qu'à faire douter de tout : elle n'a pas plutôt bâti un ouvrage, qu'elle vous montre les moiens de le ruïner. C'est une véritable Penelope, qui pendant la nuit défait la toile qu'elle avoit faite le jour. Ainsi le meilleur usage que l'on puisse faire des études de la philosophie est de connoître qu'elle est une voie d'égarement, \& que nous devons chercher un autre guide, qui est la lumiere revélée. ${ }^{4}$

Cette description du fonctionnement de la raison s'accorde avec l'interprétation de Popkin selon laquelle Bayle est un "supersceptique " : on ne peut pas avoir confiance dans les conclusions de la raison, parce que la raison elle-même peut toujours les défaire.

Elle ne fait qu'embrouiller et égarer, elle est bien inférieure au guide de la « lumière révélée ». C'est l'habituel trope fidéiste : pourquoi se contenter du guide peu fiable de la lumière naturelle quand la lumière révélée est fiable et certaine?

La formulation la plus radicale de cette conception " supersceptique » de la raison se trouve dans une remarque de l'article «Acosta (Uriel) », où Bayle utilise une analogie médicale presque effrayante pour exprimer toute l'ampleur du pouvoir destructif de la raison :

L'on peut comparer la philosophie à des poudres si corrosives qu'après avoir consumé les chairs baveuses d'une plaie, elles rongeroient la chair vive \& carieroient les os \& perceroient jusqu'aux mouelles. La philosophie réfute d'abord les erreurs ; mais, si on ne l'arrête point là, elle attaque les véritez; \& quand on la laisse faire à sa fantaisie, elle va si loin qu'elle ne sait plus où elle est, ni ne trouve plus où s'asseoir. ${ }^{5}$

En supposant que la raison soit l'instrument fondamental de la philosopheune hypothèse pertinente, dans le contexte cartésien dans lequel Bayle compose son Dictionnaire -, ce passage est un réquisitoire accablant contre la raison qui s'avère incapable de se régler. La fin naturelle de la raison, si elle n'est pas correctement limitée, est de dévorer sans distinction et la vérité et l'erreur, et de se perdre dans un bourbier intellectuel.

On aperçoit cependant, dans ce passage, les prémices d'une fonction positive de la raison. Bayle remarque que si la raison n'est pas limitée, alors 
elle devient destructrice. Ceci laisse ouverte la possibilité que la raison puisse exercer une fonction «thérapeutique», débarrasser les « plaies baveuses » des faussetés et des erreurs. La possibilité d'une fonction positive et « curative » de la raison implique que Bayle n'est pas simplement un "supersceptique » qui aurait pour but de montrer l'autocannibalisme de la raison, comme l'a soutenu Popkin. Pour que la raison fonctionne salutairement, elle a besoin de limites, et c'est cette conception critique limitée de la raison que j'attribuerai à Bayle dans ma troisième partie.

\section{II - La conception « stratonicienne » et critique de la raison}

L'autre grande interprétation plausible de la conception de la raison chez Bayle est celle d'un rationaliste critique, comme l'a proposé Gianluca Mori dans son Bayle philosophe (1999). Dans le chapitre 5, sur l'athéisme et le fidéisme, on trouve une construction de la conception baylienne de la raison fondée sur une position que Mori appelle le " stratonisme ». Bayle discute longuement cette position dans la Continuation des Pensées diverses. Straton, l'éponyme de cette position, se distinguait des autres philosophes de l'Antiquité par son athéisme absolu. Bayle s'intéresse moins à une adaptation moderne du « stratonisme ». Ce qui perdure du stratonisme ancien dans le stratonisme moderne, ce sont les thèses selon lesquelles la matière est éternelle et infinie, et que l'ordre peut exister dans la nature sans une intelligence qui la dirige. Ces deux éléments distinguent les stratoniciens des philosophes chrétiens du XVIIe siècle, quoique les uns et les autres partagent des engagements tels que le rationalisme, le mécanisme et le déterminisme (Mori, 223). Les deux thèses spécifiques des stratoniciens sont incompatibles avec le point de vue chrétien parce que l'éternité de la matière implique que, contrairement à ce que dit la Bible, la matière n'a pas été créée ex nihilo ; et la thèse de l'ordre dans une nature sans intelligence implique que, contrairement à ce qu'affirment les Écritures chrétiennes, Dieu n'est pas nécessaire pour expliquer l'ordre naturel : il n'est pas l'« auteur de la nature ». Cela conduit Mori à décrire le stratonisme de Bayle comme un « athéisme virtuel» (Mori, 222).

Mori recourt à certains textes de Bayle comme preuves qu'il fait bien un usage stratonicien de la raison. Son interprétation repose sur un trait méthodologique de la structure argumentative de Bayle. Celui-ci ne procède pas de manière directe pour soutenir sa position ; il construit la meilleure argumentation possible en faveur de la position contraire, qu'il combat par la suite. Cette défaite finale rend évidente la supériorité de la vraie position de Bayle. Il développe explicitement la position de l'athée stratonicien à 
travers plusieurs sections de la Continuation et, selon Mori, ne la réfute jamais vraiment. La position la plus fortement antagoniste de la philosophie rationnelle chrétienne se dresse toujours comme une menace qui plane sur le théisme. Le présupposé de cette interprétation est que, si sa position et sa conception de la raison n'étaient pas celles de l'athée stratonicien, le philosophe de Rotterdam aurait fourni des objections plus décisives ; étant donné l'absence de telles objections, un être rationnel est obligé d'accepter le stratonisme comme la seule position philosophique soutenable. Mori utilise l'évidence textuelle de toute la Continuation pour soutenir que la position et l'usage de la raison stratonicienne correspondent à ceux de Bayle. ${ }^{6}$

Selon cette interprétation, l'un des principes stratoniciens impliquant le plus clairement l'athéisme est que « la raison nous enseigne... que la matière est éternelle, qu'elle ne peut pas avoir un début, qu'elle est infinie » (Mori, 223). Parce que, selon Mori, le principe ex nihilo nihil fit est l'une des vérités les plus évidentes de la raison. Mori utilise ici une remarque de l'article sur Spinoza dans laquelle Bayle écrit qu' « une matière créée de rien n'est pas concevable, quelques efforts que l'on veuille faire pour se former une idée d'un acte de volonté qui convertisse en une substance réelle ce qui n'était rien auparavant. $»^{7}$

Mori se réfère également à un passage de la Réponse aux questions d'un provincial (II, 25) sur l'infinité de la matière, mais il n'est pas possible d'explorer ici ce point.

L'autre thèse stratonicienne ayant des implications athées est la négation du principe quod nescis (" ce que vous ignorez »), qui est plus ou moins le principe selon lequel seul un être intelligent peut établir et régler les lois de la nature (Mori, 223). Dans son chapitre sur Bayle et Malebranche, Mori propose une présentation plus détaillée de la manière par laquelle il pense que Bayle construit l'argument stratonicien dans la Continuation pour surmonter le principe du quod nescis. Dans ce contexte, ce principe est utilisé pour montrer qu'aucun agent doté d'une connaissance limitée (ainsi que les agents sans aucune connaissance, bien entendu) ne peut être la cause d'un univers qui dépasse de loin la connaissance humaine. Mori cite un passage de la Continuation où Bayle paraît contredire ce principe : « Il faudra donc dire qu'une nature qui existe nécessairement trouve toujours son chemin sans

6 On en trouve également des indices dans sa correspondance personnelle, dans la Réponse aux questions d'un provincial et dans d'autres textes hors de la Continuation.

7 DHC, «Spinoza », rem. O ; cité par Mori Gianluca. Bayle philosophe. Paris : Champion, 1999, p. 167. À la p. 223 n.160, Mori renvoie au chapitre sur Spinoza (p. 166ss) où il a discuté la question de la création ex nihilo et du libre arbitre divin. C'est sur ce point que porte notre propre discussion. 
qu’on le lui montre... » $(\S 114$; p. 348a). Pour Mori, ce passage montre que Bayle pourrait rejeter le quod nescis.

Selon Mori, Bayle ne répond ni aux arguments stratoniciens en faveur de l'éternité de la matière, ni à leur négation du quod nescis. Puisqu'il ne donne pas de réponse définitive à la position stratonicienne, c'est qu'il n'a pas de raison pour la rejeter. Ceci implique à mes yeux que, d'après Mori, Bayle reconnaît la supériorité de la position stratonicienne sur la philosophie chrétienne. Tout dépend donc de la question de l'approbation de l'argument et de la conception de la raison stratonicienne chez Bayle.

Puisque la position stratonicienne est une espèce d'" anti-théologie ", Mori soutient que la structure argumentative de Bayle n'exige pas que la position stratonicienne soit sans faille; il faut simplement que cette position soit rationnellement plus soutenable que la position opposée (Mori, 234-5). Un des traits caractéristiques du stratonisme, dit Mori, est l'enquête continuelle et rationnelle, ce qui permet une certaine souplesse dans la position et qui fait apparaître les revendications plus ou moins vraies, quoique faillibles (2356). Selon Mori, cette approche " probabiliste » est en définitive ce qui donne au stratonisme l'avantage sur la philosophie chrétienne ; le stratonisme ne prétend pas à la certitude de ses conclusions, mais la philosophie chrétienne est obligée de respecter les revendications de la révélation. Alors que la position stratonicienne n'est pas, à proprement parler, sceptique - puisque, comme dit Mori, le paradigme du scepticisme chez Bayle est une suspension du jugement plus typique du pyrrhonisme que du stratonisme -, le philosophe de Rotterdam emploie néanmoins une méthodologie critique et rationaliste pour parvenir à des conclusions soutenables rationnellement.

\section{Une lecture alternative de la Continuation des Pensées diverses}

L'interprétation stratonicienne dépend de l'identification de Bayle avec l'argumentation développée dans la Continuation. Pour en évaluer la pertinence, il est essentiel de resituer d'abord ces textes dans le contexte du corpus baylien, puis de considérer la signification des passages donnés dans le contexte de chaque œuvre. Une lecture attentive de la Continuation montre que Bayle garde toujours une distance critique envers la position stratonicienne, bien qu'il recoure à une conception dite «stratonicienne» de la raison.

Le prétexte de la rédaction de la Continuation a été fourni à Bayle par la réédition des Pensées diverses en 1704. Bayle réaffirme en particulier sa position des Pensées diverses selon laquelle l'idolâtrie est un mal pire que l'athéisme, et redit que les athées peuvent être moraux à cause de leur accès 
aux notions morales communes qui sont aussi au fondement de la morale chrétienne. Le contexte de la reconstruction de la position stratonicienne chez Bayle est une discussion complexe qui compare le fort et le faible des positions des philosophes païens et l'athéisme sur les questions métaphysiques et morales; bien entendu, le débat entre stratoniciens et païens est analogue au débat entre stratoniciens « modernes » du XVIIe siècle et philosophes chrétiens.

Bayle commence la présentation explicite de la position stratonicienne à la section 106. Son but est d'examiner si la technique ancienne de la rétorsion à laquelle on recourut contre les philosophes païens pourrait être utilisée par des stratoniciens «modernes» contre les philosophes chrétiens. Bayle explique d'abord la stratégie argumentative stratonicienne de la rétorsion, par laquelle on démontre que la position d'un adversaire se trouve confrontée aux mêmes difficultés que sa propre position :

Vous ne pouvez pas ignorer ce tour de l'esprit des hommes qu'après avoir embrassé une hypothese, les dificultez qui la suivent ne la leur font point quitter s'ils voient ou qu'elles leur sont communes avec leurs antagonistes, ou qu'elles ne surpassent pas les dificultez qu'ils rencontreroient ailleurs. On ne sauroit raisonnablement blâmer ceux qui ne se rendent pas à un argument qu'ils rétorquent; car tout argument qui frape le dogme de l'attaquant aussi-bien que celui du soûtenant prouve trop, \& dès là il ne prouve rien. Ce seroit donc faire le déclamateur mal à propos que de prétendre qu'un homme qui ne veut point changer d'opinion pendant que ses adversaires sont sujets aux mêmes dificultez ou à d'aussi grandes dificultez que lui, est un opiniâtre qui s'aveugle malicieusement. Son refus est très conforme aux régles de la raison. ${ }^{8}$

Bayle décrit ici le procédé de la rétorsion comme le refus de changer de position malgré des difficultés exposées par ses adversaires du fait que la position opposée présente des difficultés semblables, voire pires, que la position qu'on défend. Bayle soutient que cette stratégie est une réponse plausible d'un stratonicien ancien aux arguments téléologiques en faveur de l'existence d'une cause primaire intelligente, arguments qui utilisent l'existence de l'intention apparente et de l'ordre dans la nature comme preuves qu'il doit exister un créateur architecte de la nature :

Il n'y avoit rien, ce me semble, de plus accablant pour un philosophe stratonicien que de lui dire qu'une cause destituée de connoissance n'a point pû faire ce monde, où il y a un si bel ordre, un méchanisme si exact $\&$ des loix du mouvement si justes $\&$ si constantes. [...] Il ne faloit que cette question pour faire sentir aux stratoniciens 
que leur hypothese étoit incompréhensible \& pour les réduire à l'absurde. Il ne leur pouvoit rester que cette consolation, c'est qu'ils réduiroient au même état leurs adversaires. $^{9}$

Selon Bayle, quand le stratonicien se trouve confronté à cette objection, sa réponse ne consiste pas à démontrer qu'aucune cause intelligente n'existe, mais à démontrer que le défenseur de l'existence d'une cause intelligente rencontre des difficultés aussi nombreuses que le stratonicien, et donc que celui-ci n'a aucune raison de consentir à l'existence d'une cause intelligente. Ceci illustre la stratégie stratonicienne de la rétorsion, que Bayle trouve rationnellement défendable. Il montre plus tard dans la même section 106 comment les stratoniciens peuvent surpasser les stoïciens, les platoniciens et les péripatéticiens.

La position stratonicienne est encore l'objet de l'attention de Bayle dans la section 114, où il discute la viabilité de la doctrine cartésienne de la volonté divine libre et absolue. Bayle introduit la thèse cartésienne dans le contexte d'une discussion de la philosophie chinoise ; il dit que Dieu est la cause libre des vérités et des essences, et qu'il peut faire un cercle carré quand il lui plaît ( 114 ; p. 348a). Bayle dit aussi que la position cartésienne est très utile pour échapper aux grandes difficultés, et surtout pour rendre inutiles les chicaneries de la rétorsion des stratoniciens anciens et modernes (§114; p. 348a). Cette position cartésienne contraste avec celle de nombreux philosophes chrétiens, selon laquelle Dieu lui-même est obligé d'agir selon des vérités éternelles et nécessaires, comme les lois de la nature. Bayle concède que cette dernière position mène inévitablement au stratonisme, mais, si l'on adopte plutôt la doctrine cartésienne, les stratoniciens anciens et modernes

ne pourroient plus nous dire que nous admettons dans la Nature certaines bornes immuables qui ne dépendent point du libre arbitraire de Dieu, \& une parfaite régularité qui n'est dirigée par aucune connoissance. S'il y a des propositions d'une éternelle vérité qui sont telles de leur nature, \& non point par l'institution de Dieu, si elles ne sont point véritables par un décret libre de sa volonté, mais si au contraire il les a connuës nécessairement véritables parce que telle étoit leur nature, voilà un espece de fatum auquel il est assujeti, voilà une nécessité naturelle absolument insurmontable. ${ }^{10}$

Bayle soutient que ces dernières difficultés disparaissent si l'on affirme que les essences des êtres créés et des vérités éternelles de la raison sont fixées 
par les actes du libre arbitre de Dieu. Il termine la section 114 sur ce point, sans aller plus loin avec sa rétorsion stratonicienne, ce qui suggère qu'il pense que le stratonicien n'a pas de recours contre la thèse cartésienne.

Enfin, Bayle revisite la position stratonicienne dans les sections 149-151, où il considère le fondement de la vertu morale parmi les philosophes païens et stratoniciens. Dans ce contexte, il note que les stratoniciens soutiennent que la nature a produit une infinité d'espèces, dont chacune a des « attributs essentiels » ( $\$ 151 ;$ p. 405b). Selon la construction baylienne de la position stratonicienne, ces attributs essentiels font partie de la «nature des choses » et nous permettent de distinguer entre des espèces ainsi qu'entre le bien et le mal. ${ }^{11}$ Ces attributs essentiels servent donc à fonder la position stratonicienne de la morale. Bayle dit que, tout comme un stratonicien peut reconnaître la validité d'un syllogisme à quatre termes avec sa raison naturelle, il reconnaîtra aussi le bien et le mal avec sa raison, en les distinguant par leurs attributs essentiels. Alors qu'un stratonicien ne pourrait pas répondre à la thèse cartésienne, il semble disposer de tout ce dont il a besoin pour parvenir aux jugements moraux.

Bayle critique toutefois les fondements de ces jugements dans la section 114 à cause de la thèse cartésienne, à savoir que les essences des êtres créés sont fixés par des actes du libre arbitre de Dieu. La position stratonicienne sur la question de la régulation de la volonté dépend de l'idée que la nature elle-même fixe " des régles certaines et immuables pour... les actes de la volonté... [qui] ne sont pas toutes arbitraires : il y en a qui émanent de la nécessité de la Nature, et qui imposent une obligation indispensable ». ${ }^{12}$ Ces « règles de la volonté » sont censées ressembler aux règles de la raison - c'est-àdire de la logique - selon lesquelles on peut déterminer si la raison fonctionne correctement. En décrivant cette position, Bayle ne donne toutefois pas une explication stratonicienne de la source de ces règles de la volonté. Si agir moralement consiste à conformer la volonté à ces règles, il est essentiel que leurs fondements et leur nécessité soient bien compris.

11 L'expression « nature des choses » est assez ambiguë ; elle n'implique pas forcément l'attribution du bien et du mal aux objets ou aux événements dans le monde naturel, ou même d'un ordre particulier des objets matériaux ou des événements ; selon cette interprétation, il est possible que le bien et le mal soient attribués à quelque chose qui ressemble à un état d'affaires, un réseau complexe des relations parmi des agents, des objets, des attitudes intentionnelles, et des actions. C'est peut-être l'interprétation la plus charitable de la position, car elle évite l'attribution au stratonicien des revendications contradictoires que la matière est exempte de l'attribution morale, et que le stratonicien peut néanmoins construir des jugements moraux fondés sur la « nature des choses »; voir Bouchardy, Jean-Jacques. Pierre Bayle : la nature et «la nature des choses ». Paris: Champion, 2001, 4e partie IV, pour une discussion exhaustive des implications morales de «la nature des choses ».

12 CPD §151; OD III, p. 406a. 
L'absence d'un fondement explicite de la détermination propre de la volonté dans l'exposé de la morale stratonicienne implique que Bayle voit une sorte d'insuffisance à l'intérieur de la position stratonicienne. Bien qu'il pense que le stratonicien ait les ressources conceptuelles pour distinguer le bien du mal, celui-ci ne parvient pas à donner une explication suffisante des obligations indispensables de ces principes substantiels.

Voici encore une fois comment la thèse cartésienne du libre arbitre de Dieu pourrait donner une explication pour la normativité des thèses éthiques, bien que Bayle ne la mentionne pas explicitement dans ce contexte. Quoique la Continuation donne la description la plus complète de l'athéisme stratonicien, ce n'est pas une preuve que Bayle approuve la conception stratonicienne de la raison.

Il faut préciser que tous ces textes ne montrent pas davantage que Bayle soit cartésien ; au contraire, il y a plusieurs passages de son œuvre où il exprime ses doutes quant à la position cartésienne et à sa conception de la raison - surtout son volontarisme radical. En revanche, ce qui est clair, c'est que Bayle est loin d'affirmer l'athéisme stratonicien, et que l'interprétation de sa pensée qui déduit de ses affirmations implicites la position stratonicienne n'est pas justifiée par les textes eux-mêmes.

\section{III - La conception circonscrite et critique de la raison}

On vient de montrer que Bayle n'affirme pas la position stratonicienne. Il est clair cependant qu'il est tenté par une méthodologie stratonicienne dans son propre usage de la raison. La tactique de la rétorsion manifeste une structure semblable aux autres dispositifs de la raison dont Bayle se sert : il emploie la raison jusqu' au point où elle arrive à une impasse - soit une contradiction, soit un paradoxe dans sa propre opération - au point la raison ne peut plus fonctionner. Un exemple assez simple de ceci se trouve dans l'article « Arcésilas » :

Je veux parler de Lactance : il prétend ruïner toute la philosophie en établissant avec Socrate que l'on ne peut rien savoir, \& avec Zenon qu'il ne faut croire que ce que l'on sait. [...] Voilà de quelle maniere Lactance détruit toutes les sectes de l'ancienne philosophie les unes par les autres : 'Elles s'entr'égorgent, il n'en reste aucune en vie, dit-il : la raison en est qu'elles ont bien une épée, mais non pas un bouclier ; elles ont des forces pour les guerres offensives, mais non pas pour les défensives". [... "Arcésilas voiant cela, continue-t-il, s'arma contre toutes, \& fonda une nouvelle secte de philosophie qui consistoit à ne point philosopher." [... "Si vous prouvez que nous n'avons point de science, et qu'ainsi nous ne sommes pas philosophes, vous ne l'êtes point non plus ; car vous confessez que vous ne savez rien." [...] "Par cela même que vous ne savez aucune chose, vous en savez une." [...] Faisons quelques petites remarques sur cette dispute. [...] Le reproche de contradiction a moins de solidité que de faux brillant ; c'est plutôt une subtilité qu'une raison convaincante : le bon sens débrouille bientôt cet embaras. Si je songe que je ne dois pas croire aux songes, me voilà bien attrapé ; car si je n'y crois pas, j'y croirai ; et si j'y crois, je 
n'y croirai pas. Où est l'homme qui ne voie qu'en ce cas-là il faut excepter des autres songes celui en particulier qui m'avertit de ne croire pas aux songes ? $^{13}$

Ce passage montre que, même dans la conception la plus critique, la raison doit être circonscrite pour ne pas se cannibaliser. Bayle ici semble s'en remettre au «bon sens» pour « débrouiller » les auto-contradictions de la raison; les arguments de la raison qui mènent à l'auto-contradiction sont, d'après lui, les " subtilités ", et non une " raison convaincante ». La raison peut fonctionner efficacement aussi longtemps qu'on recourt au « bon sens » pour résoudre les difficultés où la raison produit les conclusions paradoxales ou contradictoires.

Certains interprètes soutiennent que, même avec une conception de la raison purement négative, il reste encore en elle une « capacité pour la vérité ». J.-J. Bouchardy soutient que la conscience, en particulier, préserve cette « capacité de la vérité » :

Que la raison soit « beaucoup plus propre à détruire qu'à construire », cela s'entend d'abord comme une formule d'opprobre, mais, derrière l'effet rhétorique, on ne saurait ignorer qu'une caractéristique et un usage de la raison sont signalés : elle est « beaucoup plus propre à détruire »; son rôle est rappelé, en même temps que sa limite. S'il n'y avait que ce reproche à adresser à la raison, elle serait absoute : dans un monde, dans une Nature contre-nature (contraire à la " nature des choses »), le refus et la négation peuvent être des secours et même des devoirs. [...] Ici le rôle négatif de la raison sans Dieu laisse encore une place à une capacité de vérité, si limitée et si disperse soit-elle, que l'on a déjà rencontrée - plus grande, voire intacte - dans la conscience. Peut-être ne faut-il qu'associer celle-ci à la raison. Il arrive à Bayle de les distinguer, y compris dans le Commentaire [philosophique], où il prend soin parfois d'écrire « la raison et la conscience», sans que ce soit une simple redondance. La conscience, pour ainsi dire intuitive, se fait entendre sans obliger à passer par la réflexion. Le mot « raison » est plutôt réservé à l'activité spéculative de cette faculté, au raisonnement, pour le meilleur et pour le pire. ${ }^{14}$

Bien que Bouchardy n'attribue pas à la raison une fonction positive, il admet que, lorsqu'elle est associée à la conscience, la raison a en quelque sorte une « capacité pour la vérité ». ${ }^{15}$ Bouchardy voit le « refus et la négation » de la conception de la raison baylienne comme un complément utile au rôle de la conscience qui en propose une compréhension « intuitive ». D'après cette interprétation, un modèle possible de la relation entre la raison et la conscience est que cette dernière fournit l'« intuition brute » pour la réflexion spéculative

$13 D H C$, «Arcésilas », rem. F.

14 Bouchardy, p. 198-199.

15 Je ne suis pas d'accord avec Bouchardy sur la plupart des grandes questions interprétatives, mais je trouve très utile sa glose sur l'usage de la raison chez Bayle. 
de la raison, qui peut alors prendre la décision de « refuser » ou de « nier » les données de la conscience. Bien que Bayle n'énonce pas un tel modèle dans son œuvre, il est plausible que la faculté de la raison, avec ses pouvoirs corrosifs, puisse fonctionner en tandem avec une autre faculté plus intuitive pour produire des conclusions positives.

\section{IV - Conclusion}

La conception de la raison chez Bayle est à la fois forte et faible. Elle est forte dans le sens où elle est capable d'anéantir les faussetés et les erreurs ; mais cette capacité à anéantir est aussi son talon d'Achille : laissée sans contrôle, la raison s'anéantit elle-même. Cette conception de la raison comme (auto-)destructrice exige que son pouvoir soit limité pour qu'elle fonctionne efficacement comme mise à l'épreuve des propositions vraies. Popkin accepte tout simplement que la raison soit condamnée à cet autocannibalisme, tandis que Mori voit l'athéisme stratonicien tout simplement comme la fin nécessaire de la conception de la raison. Ces deux lectures de la conception de la raison baylienne ne reconnaissent pas que Bayle envisage que la raison reçoive certaines limites : la raison fonctionne mieux quand elle opère sur les données de la conscience, l'intuition, et quand elle est utilisée avec le " bon sens », qui l'arrête dès qu'elle commence à produire les contradictions émanant d'elle-même. Cette conception de la raison, toute seule, est en fait négative et corrosive ; en revanche, quand elle est couplée avec le « bon sens », elle peut avoir un effet puissant et thérapeutique.

\section{Euvres et travaux cités}

BAYLE, Pierre. Dictionnaire historique et critique (DHC). [" cinquième » édition], 4 vol. in-folio, Amsterdam/Leyde/La Haye/Utrecht, 1740. Consultable sur internet : http://artfl-project.uchicago.edu/node/60

-, Euvres diverses, 4 vol. [reproduction en fac-similé]. Avec une introduction par E. LABROUSSE, Hildesheim : G. Olms, 1965-1982.

HUET, Pierre-Daniel. Traité philosophique de la faiblesse de l'esprit humain. Amsterdam : H. du Sauzet, 1723 ; Hildesheim : G. Olms, 1974.

BOUCHARDY, Jean-Jacques. Pierre Bayle : la nature et "la nature des choses 》. Paris : Champion, 2001.

LENNON, Thomas. Reading Bayle. Toronto : University of Toronto Press, 1999.

MORI, Gianluca. Bayle philosophe. Paris : Champion, 1999.

POPKIN, Richard. The History of Scepticism : From Savonarola to Bayle. Oxford : Oxford University Press, 2003. 